\title{
SOME RESULTS ON HYBRID RELATIVES OF THE SHEFFER POLYNOMIALS VIA OPERATIONAL RULES
}

\author{
MAHVISH ALI, TABINDA NAHID, AND SUBUHI KHAN
}

Received 07 May, 2019

\begin{abstract}
The intended objective of this paper is to introduce a new class of polynomials, namely the extended Laguerre-Gould-Hopper-Sheffer polynomials. The generating function and operational rule are derived by making use of integral transform. Their quasi-monomial properties and determinant forms are also established. Examples of certain members belonging to the extended Laguerre-Gould-Hopper-Sheffer polynomials are constructed and their corresponding results are established.
\end{abstract}

2010 Mathematics Subject Classification: 26A33; 33B10; 33C45

Keywords: Sheffer polynomials, Euler's integral, operational methods

\section{INTRODUCTION AND PRELIMINARIES}

Theory of special functions performs an essential role in the formalism of mathematical physics. They provide indeed a unique tool for developing simplified yet realistic models of physical problems, thus allowing for analytic solutions and hence a deeper insight into the problem under study. The specific physical problem indeed can suggest investigating new aspects of the well-established theory of special functions as well as introducing new family of special polynomials, which usually exhibit deeper features and thereby appear many times in new roles in numerous branches of mathematics and physics. Consequently, reformulating a physical problem in terms of special functions allows a more elegant mathematical model and then for an easier reading and numerical handling of the relevant equations as well as for the discovery of unsuspected connections with other fields of Physics.

The Sheffer polynomials are one of the most important class of polynomial sequences and have been extensively studied not only due to the fact that they arise in numerous branches of mathematics but also because of their importance in applied sciences, such as physics and engineering. In view of the result [6, p.17], the Sheffer polynomials can be defined as: 
Let $f(t)$ be a delta series and let $g(t)$ be an invertible series of the following form:

$$
f(t)=\sum_{n=0}^{\infty} f_{n} \frac{t^{n}}{n !}, \quad f_{0}=0, f_{1} \neq 0
$$

and

$$
g(t)=\sum_{n=0}^{\infty} g_{n} \frac{t^{n}}{n !}, \quad g_{0} \neq 0,
$$

then the sequences $\left\{s_{n}(x)\right\}_{n \in \mathbb{N}}$ is Sheffer for the pair $(g(t), f(t))$ if and only if the following orthogonality condition holds:

$$
\left\langle g(t) f(t)^{k} \mid s_{n}(x)\right\rangle=n ! \delta_{n, k}, \quad \forall n, k \geq 0,
$$

where $\delta_{n, k}$ is the Kronecker delta.

According to Roman [6, p.18 (Theorem 2.3.4)], the polynomial sequence $s_{n}(x)$ is uniquely determined by two (formal) power series given by equations (1.1a) and (1.1b). The exponential generating function of the Sheffer polynomials $s_{n}(x)$ is then given by

$$
\frac{1}{g\left(f^{-1}(t)\right)} \exp \left(x f^{-1}(t)\right)=\sum_{n=0}^{\infty} s_{n}(x) \frac{t^{n}}{n !},
$$

for all $x$ in $\mathbb{C}$, where $f^{-1}(t)$ is the compositional inverse of $f(t)$.

Let $\left(s_{n}(x)\right)_{n \in \mathbb{N}}$ be Sheffer sequence for $(g(t), f(t))$ satisfying the following condition:

$$
x^{n}=\sum_{k=0}^{n} a_{n, k} s_{k}(x),
$$

then $s_{n}(x)$ can be expressed by the following determinant form [9, p.232]:

$$
\begin{aligned}
& s_{0}(x)=\frac{1}{a_{0,0}}, \\
& s_{n}(x)=\frac{(-1)^{n}}{a_{0,0} a_{1,1} \cdots a_{n, n}}\left|\begin{array}{cccccc}
1 & x & x^{2} & \cdots & x^{n-1} & x^{n} \\
a_{0,0} & a_{1,0} & a_{2,0} & \cdots & a_{n-1,0} & a_{n, 0} \\
0 & a_{1,1} & a_{2,1} & \cdots & a_{n-1,1} & a_{n, 1} \\
0 & 0 & a_{2,2} & \cdots & a_{n-1,2} & a_{n, 2} \\
\cdot & \cdot & \cdot & \cdots & \cdot & \cdot \\
\cdot & \cdot & . & \cdots & \cdot & \cdot \\
0 & 0 & 0 & \cdots & a_{n-1, n-1} & a_{n, n-1}
\end{array}\right|,
\end{aligned}
$$


where $a_{n, k}$ is the $(n, k)$ entry of the Riordan array $(g(t), f(t))$ [10].

Operational methods can be exploited to simplify the derivation of properties associated with ordinary and generalized special functions and to define new families of hybrid special polynomials. The combined use of integral transforms and operational methods provides a powerful tool to deal with fractional derivatives. Using the Euler's integral [7, p.218]:

$$
a^{-v}=\frac{1}{\Gamma(v)} \int_{0}^{\infty} t^{\nu-1} e^{-a t} d t, \quad \min \{\operatorname{Re}(v), \operatorname{Re}(a)\} \geq 0,
$$

a new family of special polynomials are introduced in [1]:

$$
{ }_{L} H_{n, \nu}^{(m, r)}(x, y, z ; \alpha)=\frac{1}{\Gamma(\nu)} \int_{0}^{\infty} e^{-\alpha t} t^{\nu-1}{ }_{L} H_{n}^{(m, r)}(x, y, z, t) d t,
$$

where ${ }_{L} H_{n}^{(m, r)}(x, y, z)$ are the Laguerre-Gould-Hopper polynomials [4].

The polynomials ${ }_{L} H_{n, v}^{(m, r)}(x, y, z)$ are also defined by the following operational rule:

$$
\left(\alpha-z \frac{\partial^{r}}{\partial y^{r}}\right)^{-v} \exp \left(D_{x}^{-1} \frac{\partial^{m}}{\partial y^{m}}\right)\left\{y^{n}\right\}={ }_{L} H_{n, v}^{(m, r)}(x, y, z ; \alpha) .
$$

Most of the properties of hybrid special polynomials recognized as quasi-monomial, can be deduced by using operational rules associated with the relevant multiplicative and derivative operators. For the multi-variable hybrid special polynomials, the use of operational techniques combined with the monomiality principle provides new means of analysis for the solution of a wide class of partial differential equations often encountered in physical problems.

According to monomiality principle [2,8], a given polynomial set $r_{n}(x)(n \in$ $\mathbb{N}, x \in \mathbb{C}$ ) can be considered as quasi-monomial, if two operators $\hat{M}$ and $\hat{P}$, called "multiplicative" and "derivative" operators respectively, can be defined in such a way that

$$
\begin{aligned}
& \hat{M}\left\{r_{n}(x)\right\}=r_{n+1}(x), \\
& \hat{P}\left\{r_{n}(x)\right\}=n r_{n-1}(x),
\end{aligned}
$$

for all $n \in \mathbb{N}$. The operators $\hat{M}$ and $\hat{P}$ also satisfy the commutation relation

$$
[\hat{P}, \hat{M}]=\hat{P} \hat{M}-\hat{M} \hat{P}=\hat{1}
$$

and thus display the Weyl group structure.

If the considered polynomial set $\left\{r_{n}(x)\right\}_{n \in \mathbb{N}}$ is quasi-monomial, its properties can easily be derived from those of the $\hat{M}$ and $\hat{P}$ operators. 
If $\hat{M}$ and $\hat{P}$ have a differential realization, then

$$
\hat{M} \hat{P}\left\{r_{n}(x)\right\}=n r_{n}(x),
$$

can be interpreted as the differential equation satisfied by $r_{n}(x)$.

The theory of hybrid special polynomials has been one of the most rapidly growing research topic in mathematical analysis. In 2016, N. Raza et. al. [5] introduced the Laguerre-Gould-Hopper-Sheffer polynomials

(LGHSP) ${ }_{L} H^{(m, r)} S_{n}(x, y, z)$ which are defined by the following generating function:

$$
\begin{gathered}
\frac{1}{g\left(f^{-1}(t)\right)} C_{0}\left(-x\left(f^{-1}(t)\right)^{m}\right) \exp \left(y f^{-1}(t)+z\left(f^{-1}(t)\right)^{r}\right) \\
=\sum_{n=0}^{\infty}{ }_{L} H^{(m, r)} s_{n}(x, y, z) \frac{t^{n}}{n !} .
\end{gathered}
$$
[5]:

The following operational representations for the $\operatorname{LGHSP}_{L} H^{(m, r)} s_{n}(x, y, z)$ hold

$$
\begin{gathered}
{ }_{L} H^{(m, r)} S_{n}(x, y, z)=\exp \left(D_{x}^{-1} \frac{\partial^{m}}{\partial y^{m}}+z \frac{\partial^{r}}{\partial y^{r}}\right) s_{n}(y), \\
{ }_{L} H^{(m, r)} S_{n}(x, y, z)=\exp \left(z \frac{\partial^{r}}{\partial y^{r}}\right)_{m} L_{n}(x, y)
\end{gathered}
$$

and

$$
{ }_{L} H^{(m, r)} S_{n}(x, y, z)=\exp \left(D_{x}^{-1} \frac{\partial^{m}}{\partial y^{m}}\right) H^{(r)} S_{n}(y, z),
$$

where $s_{n}(y),{ }_{m} S_{n}(x, y)$ and $H^{(r)} S_{n}(y, z)$ are the Sheffer, 2-variable generalized Laguerre-Sheffer and Gould-Hopper-Sheffer polynomials, respectively.

For suitable choices of the variables and indices the $\operatorname{LGHSP}{ }_{L} H^{(m, r)} S_{n}(x, y, z)$ reduce to certain hybrid special polynomials. These polynomials along with their notation and name are mentioned in Table 1.

This article is an attempt to further stress the importance of the operational methods, Sheffer polynomials and Laguerre-Gould-Hopper polynomials. In Section 2, the extended Laguerre-Gould-Hopper-Sheffer polynomials are introduced by operational rule and generating function. Operational rule providing the connection between the extended Laguerre-Gould-Hopper-Sheffer polynomials and Sheffer polynomials are established. These special polynomials are framed within the context of monomiality principle formalism and their determinant form is also obtained. In the last section, the corresponding results for the extended Laguerre-Gould-Hopper-Gegenbauer polynomials and extended Laguerre-Gould-Hopper-Jacobi polynomials are derived. 
TABLE 1. Special cases of the LGHSP ${ }_{L} H^{(m, r)} S_{n}(x, y, z)$.

\begin{tabular}{|c|c|c|c|c|c|c|c|c|c|c|c|}
\hline 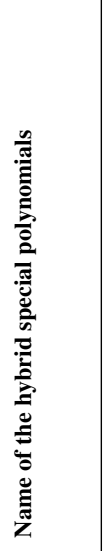 & 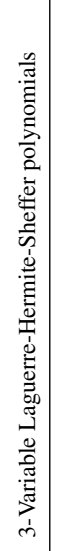 & 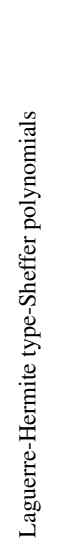 & 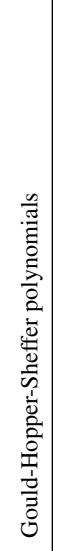 & 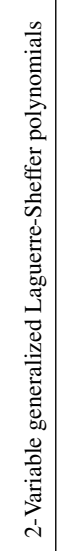 & 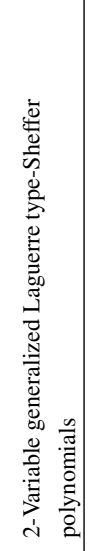 & 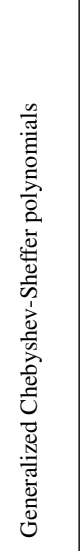 & 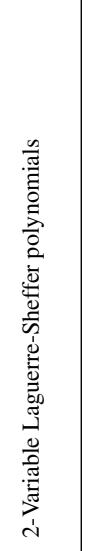 & 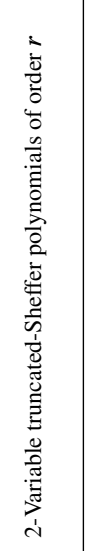 & 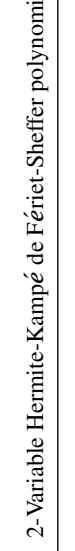 & 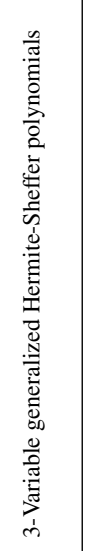 & 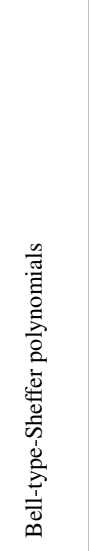 \\
\hline 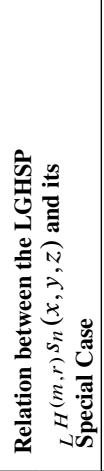 & 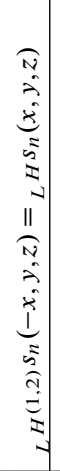 & 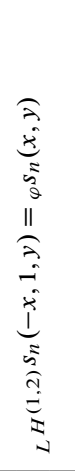 & 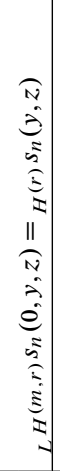 & 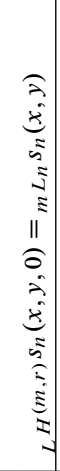 & 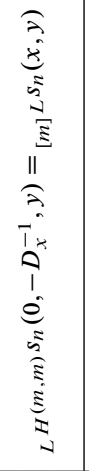 & 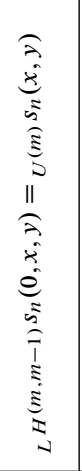 & 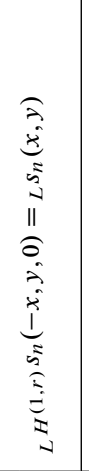 & 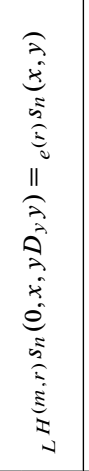 & 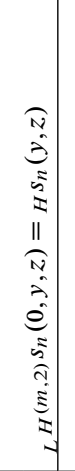 & 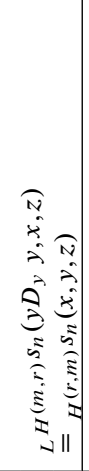 & 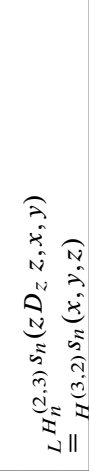 \\
\hline 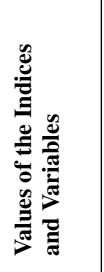 & $\begin{array}{l}\vec{u} \\
\uparrow \\
\uparrow \\
4 \\
i \\
\| \\
\vdots \\
\vec{i} \\
\| \\
\equiv\end{array}$ & 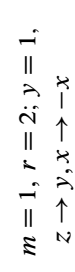 & $\begin{array}{l}0 \\
\text { II } \\
x\end{array}$ & $\begin{array}{l}0 \\
\| \\
N\end{array}$ & 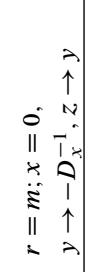 & 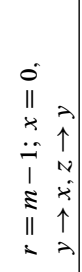 & $\begin{array}{ll}0 & \\
\| & \\
N & 4 \\
\ddot{-} & \hat{1} \\
\| & \uparrow \\
\Xi & x\end{array}$ & $\begin{array}{ll}\dot{x} & \\
\uparrow & \lambda \\
\lambda & \overrightarrow{2} \\
0 & \vec{\lambda} \\
\| & \uparrow \\
x & v\end{array}$ & $\begin{array}{l}0 \\
\| \\
x \\
\dot{H} \\
\| \\
-\end{array}$ & $\begin{array}{ll}\vec{\lambda} & \\
\overrightarrow{0} & \\
\vec{\lambda} & \vec{\lambda} \\
\uparrow & \uparrow \\
x & \lambda\end{array}$ & 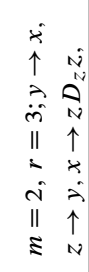 \\
\hline$\dot{\phi} \dot{\mathbf{z}}$ & $\rightarrow$ & $\dot{\theta}$ & $\dot{\Xi}$ & $\gtrsim$ & & 5 & $\dot{\vec{j}}$ & 5 & $\ddot{x}$ & & $\dot{x}$ \\
\hline
\end{tabular}

\section{EXTENDED LAGUERRE-GOULD-HOPPER-SHEFFER POLYNOMIALS}

In order to introduce the extended Laguerre-Gould-Hopper-Sheffer polynomials (ELGHSP) denoted by ${ }_{L} H^{(m, r)} S_{n, v}(x, y, z ; \alpha)$, first we prove the following theorem: 
Theorem 1. For the extended Laguerre-Gould-Hopper-Sheffer polynomials ${ }_{L} H^{(m, r)} S_{n, v}(x, y, z ; \alpha)$, the following operational rule holds true:

$$
\left(\alpha-z \frac{\partial^{r}}{\partial y^{r}}\right)^{-v}{ }_{m} L_{n}(x, y)={ }_{L} H^{(m, r)} s_{n, v}(x, y, z ; \alpha) .
$$

Proof. Replacing $a$ by $\left(\alpha-z \frac{\partial^{r}}{\partial y^{r}}\right)$ in relation (1.6) and then operating the resultant equation on 2-variable generalized Laguerre-Sheffer polynomials ${ }_{m} L s_{n}(x, y)$, it follows that

$$
\left(\alpha-z \frac{\partial^{r}}{\partial y^{r}}\right)^{-v}{ }_{m} L_{n} s_{n}(x, y)=\frac{1}{\Gamma(v)} \int_{0}^{\infty} e^{-\alpha t} t^{\nu-1} \exp \left(z t \frac{\partial^{r}}{\partial y^{r}}\right){ }_{m}{ }^{L} s_{n}(x, y) d t,
$$

which in view of equation (1.15) gives

$$
\left(\alpha-z \frac{\partial^{r}}{\partial y^{r}}\right)^{-v}{ }_{m} L_{n} s_{n}(x, y)=\frac{1}{\Gamma(\nu)} \int_{0}^{\infty} e^{-\alpha t} t^{\nu-1}{ }_{L} H^{(m, r)} s_{n}(x, y, z t) d t .
$$

Denoting the transform on the r.h.s of equation (2.3) by a new class of extended Laguerre-Gould-Hopper-Sheffer polynomials

(ELGHSP) ${ }_{L} H^{(m, r)} S_{n, v}(x, y, z ; \alpha)$, so that we have

$$
{ }_{L} H^{(m, r)} S_{n, \nu}(x, y, z ; \alpha)=\frac{1}{\Gamma(v)} \int_{0}^{\infty} e^{-\alpha t} t^{\nu-1}{ }_{L} H^{(m, r)} S_{n}(x, y, z t) d t .
$$

In view of equations (2.3) and (2.4), assertion (2.1) follows.

Next, the generating function of the ELGHSP ${ }_{L} H^{(m, r)} S_{n, v}(x, y, z ; \alpha)$ is obtained by proving the following result:

Theorem 2. The following generating function for the ELGHSP ${ }_{L} H^{(m, r)} S_{n, v}(x, y, z ; \alpha)$ holds true:

$$
\frac{\exp \left(y f^{-1}(u)\right) C_{0}\left(-x\left(f^{-1}(u)\right)^{m}\right)}{g\left(f^{-1}(u)\right)\left(\alpha-z\left(f^{-1}(u)\right)^{r}\right)^{v}}=\sum_{n=0}^{\infty}{ }_{L} H^{(m, r)} S_{n, v}(x, y, z ; \alpha) \frac{u^{n}}{n !} .
$$

Proof. Multiplying on both sides of equation (2.4) by $\frac{u^{n}}{n !}$ and summing over $n$, we find

$$
\sum_{n=0}^{\infty}{ }_{L} H^{(m, r)} S_{n, v}(x, y, z ; \alpha) \frac{u^{n}}{n !}=\sum_{n=0}^{\infty} \frac{1}{\Gamma(\nu)} \int_{0}^{\infty} e^{-\alpha t} t^{\nu-1}{ }_{L} H^{(m, r)} S_{n}(x, y, z t) \frac{u^{n}}{n !} d t,
$$


which on using equation (1.13) in the r.h.s. gives

$$
\begin{gathered}
\sum_{n=0}^{\infty}{ }_{L} H^{(m, r)} S_{n, v}(x, y, z ; \alpha) \frac{u^{n}}{n !} \\
=\frac{C_{0}\left(-x\left(f^{-1}(u)\right)^{m}\right) \exp \left(y f^{-1}(u)\right)}{g\left(f^{-1}(u)\right) \Gamma(v)} \int_{0}^{\infty} e^{-\left(\alpha-z\left(f^{-1}(u)\right)^{r}\right) t} t^{\nu-1} d t .
\end{gathered}
$$

Making use of equation (1.6) in the r.h.s. of equation (2.7), yields assertion (2.5).

Remark 1. For $\alpha=1, v=1$ and $z=D_{z}^{-1}$, the extended Laguerre-Gould-HopperSheffer polynomials ${ }_{L} H^{(m, r)} S_{n, v}(x, y, z ; \alpha)$ reduce to the Laguerre-Gould-HopperSheffer polynomials ${ }_{L} H^{(m, r)} S_{n}(x, y, z)[5]$.

Differentiating generating function (2.5) w.r.t $\alpha$, the following recurrence relation for the ELGHSP ${ }_{L} H^{(m, r)} S_{n, v}(x, y, z ; \alpha)$ is obtained:

$$
\frac{\partial}{\partial \alpha}{ }_{L} H^{(m, r)} S_{n, v}(x, y, z ; \alpha)=-v_{L} H^{(m, r)} S_{n, v+1}(x, y, z ; \alpha) .
$$

In order to derive quasi-monomial properties and operational representation for the ELGHSP ${ }_{L} H^{(m, r)} S_{n, v}(x, y, z ; \alpha)$, the following operation will be used:

$(\Theta)$ : Replacement of $z$ by $z t$, multiplication by $\frac{1}{\Gamma(v)} e^{-\alpha t} t^{\nu-1}$ and then integration with respect to $t$ from $t=0$ to $t=\infty$.

To frame the ELGHSP ${ }_{L} H^{(m, r)} S_{n, v}(x, y, z ; \alpha)$ within the context of monomiality principle, we prove the following result:

Theorem 3. The ELGHSP ${ }_{L} H^{(m, r)} S_{n, v}(x, y, z ; \alpha)$ are quasi-monomial with respect to the following multiplicative and derivative operators:

$$
\hat{M}_{L H s_{v}}=\left(y+m D_{x}^{-1} \frac{\partial^{m-1}}{\partial y^{m-1}}-r z \frac{\partial^{r}}{\partial \alpha \partial y^{r-1}}-\frac{g^{\prime}\left(\partial_{y}\right)}{g\left(\partial_{y}\right)}\right) \frac{1}{f^{\prime}\left(\partial_{y}\right)}
$$

and

respectively, where $\partial_{y}:=\frac{\partial}{\partial y}$.

$$
\hat{P}_{L H s_{v}}=f\left(\partial_{y}\right),
$$

Proof. We recall the following equations [5]:

$$
\begin{gathered}
\left(y+m D_{x}^{-1} \frac{\partial^{m-1}}{\partial y^{m-1}}+r z \frac{\partial^{r-1}}{\partial y^{r-1}}-\frac{g^{\prime}\left(\partial_{y}\right)}{g\left(\partial_{y}\right)}\right) \frac{1}{f^{\prime}\left(\partial_{y}\right)}{ }_{L} H^{(m, r)} S_{n}(x, y, z) \\
={ }_{L} H^{(m, r)} S_{n+1}(x, y, z) ;
\end{gathered}
$$




$$
f\left(\partial_{y}\right)_{L} H^{(m, r)} S_{n}(x, y, z)=n_{L} H^{(m, r)} S_{n-1}(x, y, z) .
$$

Performing the operation $(\Theta)$ on equations (2.11) and (2.12) and using recurrence relation (2.8), we obtain assertions (2.9) and (2.10).

The following corollary is an immediate consequence of Theorem 3:

Corollary 1. The following differential equation for the ELGHSP ${ }_{L} H^{(m, r)} S_{n, v}(x, y, z ; \alpha)$ holds true:

$$
\left(\left(y+m D_{x}^{-1} \frac{\partial^{m-1}}{\partial y^{m-1}}-r z \frac{\partial^{r}}{\partial \alpha \partial y^{r-1}}-\frac{g^{\prime}\left(\partial_{y}\right)}{g\left(\partial_{y}\right)}\right) \frac{f\left(\partial_{y}\right)}{f^{\prime}\left(\partial_{y}\right)}-n\right){ }_{L} H^{(m, r)} s_{n, v}(x, y, z ; \alpha)=0
$$

Proof. Using equations (2.9) and (2.10) in equation (1.12), assertion (2.13) follows.

The operational representation between the ELGHSP ${ }_{L} H^{(m, r)} S_{n, v}(x, y, z ; \alpha)$ and Sheffer polynomials $s_{n}(x)$ is obtained in the form of the following result:

Theorem 4. The following operational representation between the ELGHSP ${ }_{L} H^{(m, r)} S_{n, v}(x, y, z ; \alpha)$ and Sheffer polynomials $s_{n}(x)$ holds true:

$$
\left(\alpha-z \frac{\partial^{r}}{\partial y^{r}}\right)^{-v} \exp \left(D_{x}^{-1} \frac{\partial^{m}}{\partial y^{m}}\right)\left\{s_{n}(y)\right\}={ }_{L} H^{(m, r)} s_{n, v}(x, y, z ; \alpha) .
$$

Proof. Performing operation $(\Theta)$ on equation (1.14), we obtain

$$
\begin{gathered}
\frac{1}{\Gamma(\nu)} \int_{0}^{\infty} e^{-\alpha t} t^{\nu-1} \exp \left(D_{x}^{-1} \frac{\partial^{m}}{\partial y^{m}}+z t \frac{\partial^{r}}{\partial y^{r}}\right) s_{n}(y) d t \\
=\frac{1}{\Gamma(\nu)} \int_{0}^{\infty} e^{-\alpha t} t^{\nu-1}{ }_{L} H^{(m, r)} s_{n}(x, y, z t) d t .
\end{gathered}
$$

Decoupling the exponential operator in the 1.h.s. of above equation by using the Weyl identity [3, p. 7]

$$
e^{\hat{A}+\hat{B}}=e^{-k / 2} e^{\hat{A}} e^{\hat{B}}, \quad k \in \mathbb{C},
$$

we get

$$
\exp \left(D_{x}^{-1} \frac{\partial^{m}}{\partial y^{m}}\right) \frac{1}{\Gamma(\nu)} \int_{0}^{\infty} e^{-\left(\alpha-z \partial^{r}{ }^{r}\right) t} t^{\nu-1} d t s_{n}(y)={ }_{L} H^{(m, r)} S_{n, v}(x, y, z ; \alpha),
$$

which in view of relation (1.6), yields assertion (2.14). 
The determinant definition of the Sheffer sequences proposed by W. Wang [9] in 2014, provides motivation to establish the determinant forms of the new hybrid special polynomials. The determinant approach is equivalent to the corresponding approach based on operational methods. This approach is beneficial in detecting the solution of general linear interpolation problems and also suitable for computations. Inspired by the novel work on determinant approaches, the determinant definition of the ELGHSP ${ }_{L} H^{(m, r)} S_{n, v}(x, y, z ; \alpha)$ is established by proving the following result:

Theorem 5. The ELGHSP ${ }_{L} H^{(m, r)} S_{n, v}(x, y, z ; \alpha)$ of degree $n$ are defined by

$$
\begin{aligned}
& { }_{L} H^{(m, r)} S_{0, v}(x, y, z ; \alpha)=\frac{1}{a_{0,0}} L_{0, v} H_{0, r)}^{(m, x, y, z ; \alpha),} \\
& { }_{L} H^{(m, r)} S_{n, v}(x, y, z ; \alpha)=\frac{(-1)^{n}}{a_{0,0} a_{1,1} \ldots a_{n, n}} \\
& \times\left|\begin{array}{ccccc}
{ }_{L} H_{0, v}^{(m, r)}(x, y, z ; \alpha) & { }_{L} H_{1, v}^{(m, r)}(x, y, z ; \alpha) & \cdots & { }_{L} H_{n-1, v}^{(m, r)}(x, y, z ; \alpha) & { }_{L} H_{n, v}^{(m, r)}(x, y, z ; \alpha) \\
a_{0,0} & a_{1,0} & \cdots & a_{n-1,0} & a_{n, 0} \\
0 & a_{1,1} & \cdots & a_{n-1,1} & a_{n, 1} \\
0 & 0 & \cdots & a_{n-1,2} & a_{n, 2} \\
\cdot & \cdot & \cdots & \cdot & \cdot \\
0 & \cdot & \cdots & \cdot & a_{n, n-1}
\end{array}\right|,
\end{aligned}
$$

where $a_{n, k}$ is the $(n, k)$ entry of the Riordan array $(g(t), f(t))$.

Proof. Interchanging $x$ by $y$ and operating $\left(\alpha-z \frac{\partial^{r}}{\partial y^{r}}\right)^{-v} \exp \left(D_{x}^{-1} \frac{\partial^{m}}{\partial y^{m}}\right)$ on both sides of equation (1.4) and using operational rules (1.8) and (2.14) in r.h.s and l.h.s. respectively, we find

$$
{ }_{L} H_{n, v}^{(m, r)}(x, y, z ; \alpha)=\sum_{k=0}^{n} a_{n, k}{ }_{L} H^{(m, r)} s_{n, v}(x, y, z ; \alpha)
$$

The above equality leads to the following system of infinite equations in the unknowns ${ }_{L} H^{(m, r)} S_{n, v}(x, y, z ; \alpha), \quad n=0,1, \ldots$, 


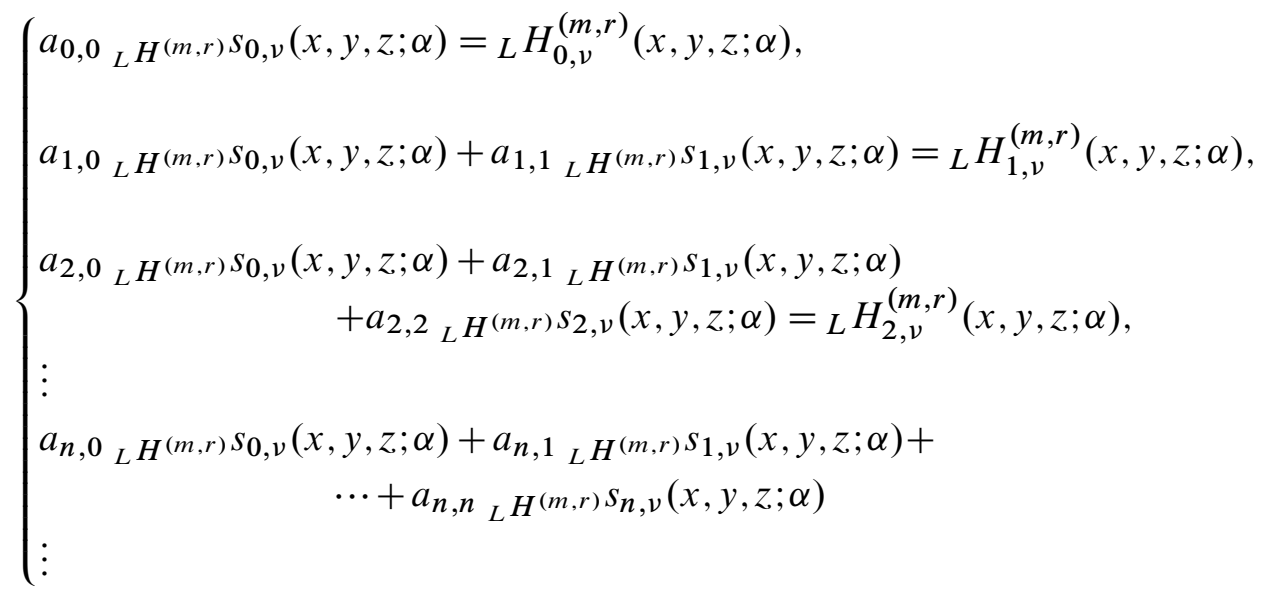

From first equation of system (2.21), assertion (2.18) follows. Applying the Cramer's rule to the first $n+1$ equations, it follows that

$$
=\frac{{ }_{L} H^{(m, r)} S_{n, v}(x, y, z ; \alpha)}{a_{0,0} a_{1,1} \ldots a_{n, n}}\left|\begin{array}{ccccc}
a_{0,0} & 0 & \ldots & 0 & L_{L} H_{0, v}^{(m, r)}(x, y, z ; \alpha) \\
a_{1,0} & a_{1,1} & \ldots & 0 & L_{L} H_{1, v}^{(m, r)}(x, y, z ; \alpha) \\
a_{2,0} & a_{2,1} & \cdots & 0 & L_{2, v} H^{(m, r)}(x, y, z ; \alpha) \\
\cdot & . & \cdots & . & \\
a_{n-1,0} & a_{n-1,1} & \cdots & a_{n-1, n-1} & { }_{L} H_{n-1, v}^{(m, r)}(x, y, z ; \alpha) \\
a_{n, 0} & a_{n, 1} & \cdots & a_{n, n-1} & { }_{L} H_{n, v}^{(m, r)}(x, y, z ; \alpha)
\end{array}\right| .
$$

Now, bringing the $(n+1)$-th column to the first place by $n$ transpositions of adjacent columns and noting that the determinant of a square matrix is the same as that of its transpose, assertion (2.19) follows.

In the next section, certain members belonging to the ELGHSP ${ }_{L} H^{(m, r)} S_{n, v}(x, y, z ; \alpha)$ are constructed and their analogues results are established. 


\section{EXAMPLES}

The Sheffer family contains several polynomials as their members for different choices of $g(t)$ and $f(t)$. Thus, by taking $g(t)$ and $f(t)$ of the special polynomials belonging to Sheffer family, we get the corresponding members belonging to the ELGHSP family ${ }_{L} H^{(m, r)} S_{n, v}(x, y, z ; \alpha)$.

Example 3.1 Taking $g(u)=\left(\frac{2}{1+\sqrt{1-u^{2}}}\right)^{\lambda}, f(u)=\frac{-u}{1+\sqrt{1-u^{2}}}, f^{-1}(u)=\frac{-2 u}{1+u^{2}}$ and $g\left(f^{-1}(u)\right)=\left(1+u^{2}\right)^{\lambda}$ (for which the Sheffer polynomials becomes the Gegenbauer polynomials $C_{n}^{(\lambda)}(x)$ [9]) in Theorem 2- Theorem 4, we find that:

The following generating function for the extended Laguerre-Gould-Hopper Gegenbauer polynomials (ELGHGnP) ${ }_{L} H^{(m, r)} C_{n, \nu}^{(\lambda)}(x, y, z ; \alpha)$ holds true:

$$
\frac{\exp \left(y\left(\frac{-2 u}{1+u^{2}}\right)\right) C_{0}\left(-x\left(\frac{-2 u}{1+u^{2}}\right)^{m}\right)}{\left(1+u^{2}\right)^{\lambda}\left(\alpha-z\left(\frac{-2 u}{1+u^{2}}\right)^{r}\right)^{v}}=\sum_{n=0}^{\infty}{ }_{L} H^{(m, r)} C_{n, \nu}^{(\lambda)}(x, y, z ; \alpha) \frac{u^{n}}{n !} .
$$

The ELGHGnP ${ }_{L} H^{(m, r)} C_{n, \nu}^{(\lambda)}(x, y, z ; \alpha)$ are quasi-monomial with respect to the following multiplicative and derivative operators:

$$
\hat{M}_{L H C_{v}}=\lambda \partial y+\left(y+m D_{x}^{-1} \frac{\partial^{m-1}}{\partial y^{m-1}}-r z \frac{\partial^{r}}{\partial \alpha \partial y^{r-1}}\right)\left((\partial y)^{2}-1-\sqrt{1-(\partial y)^{2}}\right),
$$

and

$$
\hat{P}_{L H C_{v}}=\frac{-\partial y}{1+\sqrt{1-(\partial y)^{2}}} .
$$
tion:

The ELGHGnP ${ }_{L} H^{(m, r)} C_{n, v}^{(\lambda)}(x, y, z ; \alpha)$ satisfy the following differential equa-

$$
\begin{gathered}
\left(\frac{-\lambda(\partial y)^{2}}{1+\sqrt{1-(\partial y)^{2}}}+\left(y \frac{\partial}{\partial y}+m D_{x}^{-1} \frac{\partial^{m}}{\partial y^{m}}-r z \frac{\partial^{r+1}}{\partial \alpha \partial y^{r}}\right) \frac{\left(1-(\partial y)^{2}+\sqrt{1-(\partial y)^{2}}\right)}{1+\sqrt{1-(\partial y)^{2}}}-n\right) \\
\times_{L^{(m, r)}} C_{n, \nu}^{(\lambda)}(x, y, z ; \alpha)=0 .
\end{gathered}
$$

The following relation between the Gegenbauer polynomials $C_{n}^{(\lambda)}(x)$ and ELGHGnP ${ }_{L} H^{(m, r)} C_{n, v}^{(\lambda)}(x, y, z ; \alpha)$ holds true:

$$
\left(\alpha-z \frac{\partial^{r}}{\partial y^{r}}\right)^{-v} \exp \left(D_{x}^{-1} \frac{\partial^{m}}{\partial y^{m}}\right)\left\{C_{n}^{(\lambda)}(y)\right\}={ }_{L} H^{(m, r)} C_{n, \nu}^{(\lambda)}(x, y, z ; \alpha) .
$$


Taking

$$
a_{n, k}= \begin{cases}0, & \text { if } n-k \text { is odd, } \\
\frac{c_{n}(-1)^{k}(\lambda+k)}{c_{k} 2^{n}(\lambda+n)}\left(\begin{array}{c}
\lambda+n \\
n-k / 2
\end{array}\right), & \text { if } n-k \text { is even, }\end{cases}
$$

where $c_{n}=1 /\left(\begin{array}{c}-\lambda \\ n\end{array}\right)$ and $a_{n, n}=\left(-\frac{1}{2}\right)^{n}$ in equations (2.18) and (2.19), we find that the ELGHGnP ${ }_{L} H^{(m, r)} C_{n, v}^{(\lambda)}(x, y, z ; \alpha)$ for $n=4$ are defined by

$$
\begin{aligned}
& { }_{L} H^{(m, r)} C_{0, v}^{(\lambda)}(x, y, z ; \alpha)={ }_{L} H_{0, v}^{(m, r)}(x, y, z ; \alpha), \\
& { }_{L} H^{(m, r)} C_{n, \nu}^{(\lambda)}(x, y, z ; \alpha)=\frac{128 \lambda(\lambda+1)(\lambda+2)(\lambda+3)}{3} \\
& { }_{L} H_{0, v}^{(m, r)}(x, y, z ; \alpha) \quad{ }_{L} H_{1, v}^{(m, r)}(x, y, z ; \alpha) \quad{ }_{L} H_{2, v}^{(m, r)}(x, y, z ; \alpha) \quad{ }_{L} H_{3, v}^{(m, r)}(x, y, z ; \alpha) \quad{ }_{L} H_{4, v}^{(m, r)}(x, y, z ; \alpha) \\
& \begin{array}{ccccc}
1 & 0 & \frac{1}{2(\lambda+1)} & 0 & \frac{3}{4(\lambda+1)(\lambda+2)} \\
0 & -\frac{1}{2} & 0 & \frac{-3}{4(\lambda+2)} & 0 \\
0 & 0 & \frac{1}{4} & 0 & \frac{3}{4(\lambda+3)} \\
0 & 0 & 0 & -\frac{1}{8} & 0
\end{array} \\
& \times
\end{aligned}
$$

Example 3.2 Taking $g(u)=\left(\frac{2}{1+\sqrt{1+2 u}}\right)^{1+\alpha+\beta}, f(u)=\frac{u}{1+u+\sqrt{1+2 u}}, f^{-1}(u)=$ $\frac{2 u}{(1-u)^{2}}$ and

$g\left(f^{-1}(u)\right)=(1-u)^{1+\alpha+\beta}$ (for which the Sheffer polynomials becomes the Jacobi polynomials $J_{n}(x)$ [9]) in Theorem 2 - Theorem 4, we find that:

The following generating function for the extended Laguerre-Gould-Hopper-Jacobi polynomials (ELGHJP) ${ }_{L} H^{(m, r)} J_{n, v}(x, y, z ; \alpha)$ holds true:

$$
\frac{\exp \left(y\left(\frac{2 u}{(1-u)^{2}}\right)\right) C_{0}\left(-x\left(\frac{2 u}{(1-u)^{2}}\right)^{m}\right)}{(1-u)^{1+\alpha+\beta}\left(\alpha-z\left(\frac{2 u}{(1-u)^{2}}\right)^{r}\right)^{v}}=\sum_{n=0}^{\infty}{ }_{L} H^{(m, r)} J_{n, v}(x, y, z ; \alpha) \frac{u^{n}}{n !} .
$$

The ELGHJP ${ }_{L} H^{(m, r)} J_{n, v}(x, y, z ; \alpha)$ are quasi-monomial with respect to the following multiplicative and derivative operators:

$$
\begin{aligned}
\hat{M}_{L H J_{v}}= & \left(y+m D_{x}^{-1} \frac{\partial^{m-1}}{\partial y^{m-1}}-r z \frac{\partial^{r}}{\partial \alpha \partial y^{r-1}}+\frac{(1+\alpha+\beta)}{1+2 \partial y+\sqrt{1+2 \partial y}}\right) \\
& \times(1+\partial y+\sqrt{1+2 \partial y}) \sqrt{1+2 \partial y}
\end{aligned}
$$

and

$$
\hat{P}_{L H J_{v}}=\frac{\partial y}{1+\partial y+\sqrt{1+2 \partial y}} .
$$


The ELGHJP ${ }_{L} H^{(m, r)} J_{n, v}(x, y, z ; \alpha)$ satisfy the following differential equation:

$$
\begin{gathered}
\left(\left(y \frac{\partial}{\partial y}+m D_{x}^{-1} \frac{\partial^{m}}{\partial y^{m}}-r z \frac{\partial^{r+1}}{\partial y^{r} \partial \alpha}-\frac{(1+\alpha+\beta) \partial y}{1+2 \partial y+\sqrt{1+2 \partial y}}\right) \sqrt{1+2 \partial y}-n\right) \\
\times{ }_{L} H^{(m, r)} J_{n, v}(x, y, z ; \alpha)=0 .
\end{gathered}
$$

The following relation between the Jacobi polynomials $J_{n}(x)$ and ELGHJP $_{L} H^{(m, r)} J_{n, v}(x, y, z ; \alpha)$ holds true:

$$
\left(\alpha-z \frac{\partial^{r}}{\partial y^{r}}\right)^{-v} \exp \left(D_{x}^{-1} \frac{\partial^{m}}{\partial y^{m}}\right)\left\{J_{n}(y)\right\}={ }_{L} H^{(m, r)} J_{n, v}(x, y, z ; \alpha) .
$$

Taking

$$
a_{n, k}=\frac{(-1)^{n-k}}{2^{n}} \frac{c_{n}}{c_{k}} \frac{1+\alpha+\beta+2 k}{1+\alpha+\beta+2 n}\left(\begin{array}{c}
1+\alpha+\beta+2 n \\
n-k
\end{array}\right),
$$

where $c_{n}=\frac{4^{n}(\alpha+n)_{n} n !}{(\alpha+\beta+2 n)_{2 n}}$ and $a_{n, n}=\left(\frac{1}{2}\right)^{n}$ in equations (2.18) and (2.19), we find that the ELGHJP ${ }_{L} H^{(m, r)} J_{n, v}(x, y, z ; \alpha)$ for $n=4$ are defined by

$$
\begin{aligned}
& { }_{L} H^{(m, r)} J_{0, v}(x, y, z ; \alpha)={ }_{L} H_{0, v}^{(m, r)}(x, y, z ; \alpha), \\
& { }_{L} H^{(m, r)} J_{n, v}(x, y, z ; \alpha)=1024 \\
& \left.\times \mid \begin{array}{ccccc}
{ }_{L} H_{0, v}^{(m, r)}(x, y, z ; \alpha) & { }_{L} H_{1, v}^{(m, r)}(x, y, z ; \alpha) & { }_{L} H_{2, \nu}^{(m, r)}(x, y, z ; \alpha) & { }_{L} H_{3, v}^{(m, r)}(x, y, z ; \alpha) & { }_{L} H_{4, v}^{(m, r)}(x, y, z ; \alpha) \\
1 & -\frac{2(\alpha+1)}{\alpha+\beta+2} & \frac{4(\alpha+2))_{2}}{(\alpha+\beta+3)_{2}} & -\frac{8(\alpha+3)_{3}}{(\alpha+\beta+4)_{3}} & \frac{16(\alpha+4)_{4}}{(\alpha+\beta+5)_{4}} \\
0 & \frac{1}{2} & -\frac{2(\alpha+2)}{\alpha+\beta+4} & \frac{6(\alpha+3) 2}{(\alpha+\beta+5)_{2}} & -\frac{16(\alpha+)_{3}}{(\alpha+\beta+)_{3}} \\
0 & 0 & \frac{1}{4} & -\frac{3(\alpha+3)}{2(\alpha+\beta+6)} & \frac{6(\alpha+4)_{2}}{(\alpha+\beta+7)_{2}} \\
0 & 0 & 0 & \frac{1}{8} & -\frac{\alpha+4}{\alpha+\beta+8}
\end{array}\right] .
\end{aligned}
$$

The above examples show that the operational rules provide a mechanism to obtain the results for the members belonging to the ELGHSP ${ }_{L} H^{(m, r)} S_{n, v}(x, y, z ; \alpha)$ and prove the usefulness of the method adopted in this paper. The operational techniques can be used for a more general insight into the theory of hybrid special polynomials and for their extensions. The appropriate combination of methods relevant to generalized operational calculus and to special functions can be very useful tool to treat a large body of problems both in physics and mathematics. Thus, we conclude that the method based on the operational rules may provide powerful tools to deal with the possibilities offered by extended forms of the hybrid special polynomials. 


\section{APPENDIX}

We have mentioned several special cases of the $\operatorname{LGHSP}_{L} H^{(m, r)} S_{n}(x, y, z)$ in Table 1. Now, for the same choice of the variables and indices, the ELGHSP ${ }_{L} H^{(m, r)} S_{n, v}(x, y, z ; \alpha)$ reduce to the corresponding special case. These new hybrid special polynomials related to the Sheffer polynomials are given in Table 2.

TABLE 2. Special cases of the ELGHSP ${ }_{L} H^{(m, r)} S_{n, v}(x, y, z ; \alpha)$.

\begin{tabular}{|c|c|c|c|c|c|c|c|c|c|c|c|}
\hline 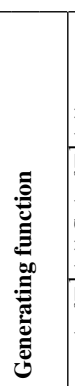 & 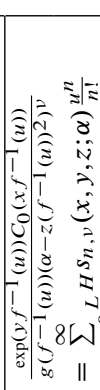 & 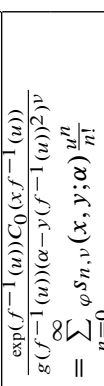 & 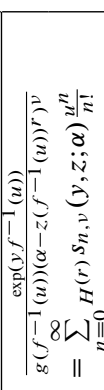 & 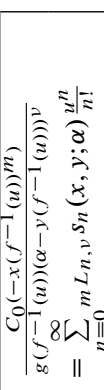 & 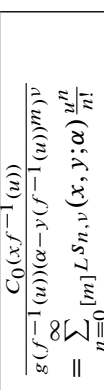 & 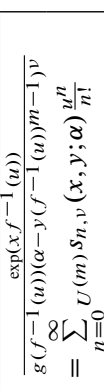 & 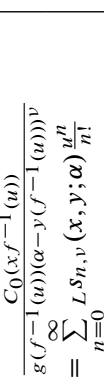 & 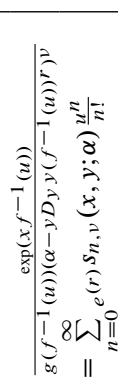 & 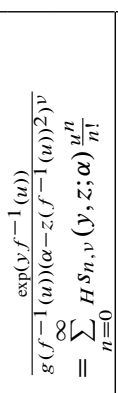 & 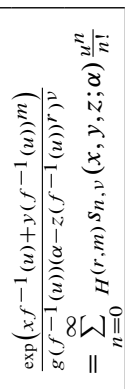 & 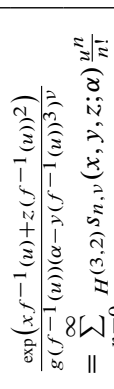 \\
\hline 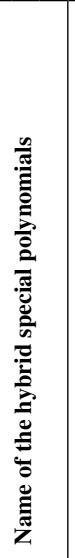 & 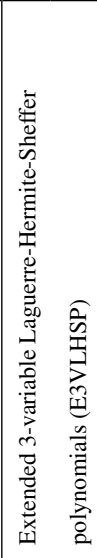 & 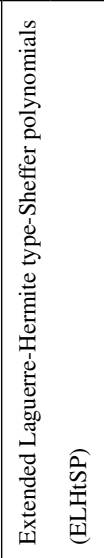 & 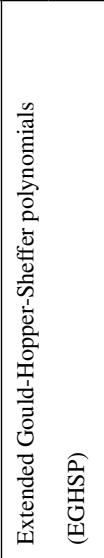 & 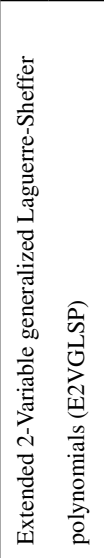 & 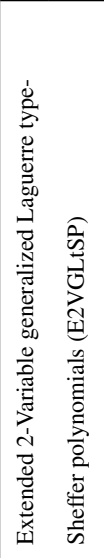 & 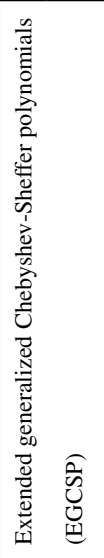 & 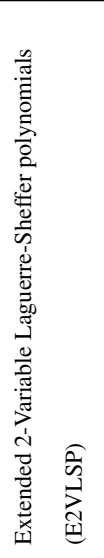 & 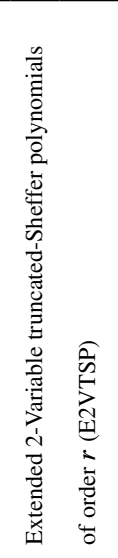 & 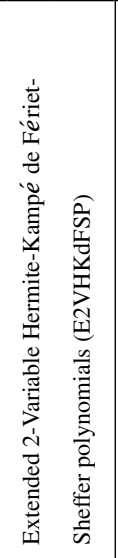 & 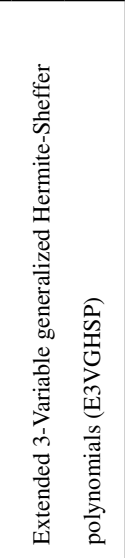 & 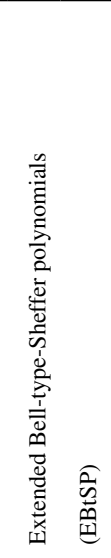 \\
\hline 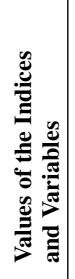 & 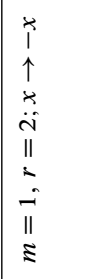 & 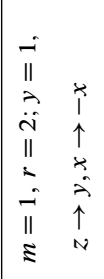 & $\begin{array}{l}0 \\
\text { II } \\
x\end{array}$ & $\begin{array}{ll}0 & \\
\| & \\
\vec{y} & \\
\vec{\theta} & \overrightarrow{1} \\
- & N\end{array}$ & 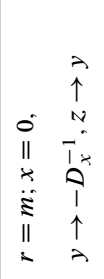 & 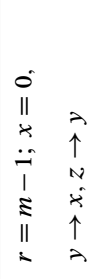 & $\begin{array}{ll} & \\
\| & \\
N & \\
\ddot{Z} & \hat{1} \\
\| & \uparrow \\
\Xi & x\end{array}$ & 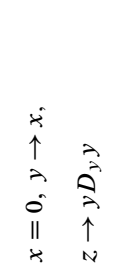 & $\begin{array}{l}0 \\
11 \\
x \\
i \\
\text { II } \\
-\end{array}$ & $\begin{array}{ll}\vec{\lambda} & \\
\hat{\lambda} & \\
\lambda & x \\
\uparrow & \uparrow \\
x & \lambda\end{array}$ & $\begin{array}{l}\vec{x} \\
\uparrow \\
\vec{n} \\
\| \\
\vdots \\
\bar{N} \\
\| \\
\equiv \\
\equiv\end{array}$ \\
\hline$\dot{m} \dot{z}$ & - & $\dot{A}$ & $\dot{\Xi}$ & $z$ & $>$ & $>$ & 5 & $\dot{\bar{F}}$ & $\ddot{凶}$ & $\dot{x}$ & $\dot{x}$ \\
\hline
\end{tabular}




\section{ACKNOWLEDGEMENT}

This work has been done under Dr. D. S. Kothari Post Doctoral Fellowship (Award letter No. F.4-2/2006(BSR)/MA/17-18/0025) awarded to Dr. Mahvish Ali and Senior Research Fellowship (Award letter No. F./2014-15/NFO-2014-15-OBC-UTT24168/(SA-III/Website)) awarded to Ms. Tabinda Nahid by the University Grants Commission, Government of India, New Delhi.

\section{REFERENCES}

[1] A. A. Al-Gonah, "Some results involving special polynomials and integral transforms." Palestine Journal of Mathematics., vol. 4, no. 2, pp. 327-334, 2015.

[2] G. Dattoli, "Hermite-Bessel and Laguerre-Bessel functions: A by-product of the monomiality principle." Proc. Melfi Sch. Adv. Top. Math. Phys., vol. 1, pp. 147-164, 2000.

[3] G. Dattoli, P. L. Ottaviani, A. Torre, and L. Vázquez, "Evolution operator equations: integration with algebraic and finite-difference methods. Applications to physical problems in classical and quantum mechanics and quantum field theory." Riv. Nuovo Cimento Soc. Ital. Fis., vol. 20, no. 2, pp. 1-133, 1997.

[4] S. Khan and A. A. Al-Gonah, "Operational methods and Laguerre-Gould Hopper polynomials.” Appl. Math. Comput., vol. 218, pp. 9930-9942, 2012, doi: https://doi.org/10.1016/j.amc.2012.03.080.

[5] N. Raza, S. Khan, and M. Ali, "Properties of certain new special polynomials associated with Sheffer sequences." Tbilisi Mathematical Journal , vol. 9, no. 1, pp. 245-270, 2016, doi: https://doi.org/10.1515/tmj-2016-001.

[6] S. Roman, The Umbral Calculus. New York: Academic Press., 1984.

[7] H. M. Srivastava and H. L. Manocha, A Treatise on Generating Functions. New York: Halsted Press., 1984.

[8] J. F. Steffensen, " The poweroid, an extension of the mathematical notion of power." Acta. Math., vol. 73, pp. 333-366, 1941.

[9] W. Wang, “A determinantal approach to Sheffer sequences." Linear Algebra Appl., vol. 463, pp. 228-254, 2014.

[10] W. Wang and T. Wang, “ Generalized Riordan arrays.” Discrete Math., vol. 308, pp. 6466-6500, 2008 .

Authors' addresses

\section{Mahvish Ali}

Department of Applied Sciences and Humanities, Faculty of Engineering and Technology, Jamia Millia Islamia (A Central University), New Delhi-110025, India,

E-mail address: mahvishali37@gmail.com

\section{Tabinda Nahid}

Department of Mathematics, Aligarh Muslim University, Aligarh, India

E-mail address: tabindanahidegmail.com

\section{Subuhi Khan}

Department of Mathematics, Aligarh Muslim University, Aligarh, India

E-mail address: subuhi2006@gmail.com 